\title{
Toward an Automatic Calibration of Dual Fluoroscopy Imaging Systems
}

\author{
Kaleel Al-Durgham, Derek Lichti, Gregor Kuntze*, Gulshan Sharma*, Janet Ronsky*
}

\author{
Departments of Geomatics Engineering and *Mechanical \& Manufacturing Engineering, University of Calgary, Calgary, Alberta, \\ Canada T2N $1 \mathrm{~N} 4$ \\ (kmaldurg, ddlichti, gkuntze, gbsharma, jlronsky @ucalgary.ca)
}

Commission V, WG V/5

KEY WORDS: Dual Fluoroscopy, Biplanar Videoradiography, Photogrammetric Calibration, Target Extraction

\begin{abstract}
:
High-speed dual fluoroscopy (DF) imaging provides a novel, in-vivo solution to quantify the six-degree-of-freedom skeletal kinematics of humans and animals with sub-millimetre accuracy and high temporal resolution. A rigorous geometric calibration of DF system parameters is essential to ensure precise bony rotation and translation measurements. One way to achieve the system calibration is by performing a bundle adjustment with self-calibration. A first-time bundle adjustment-based system calibration was recently achieved. The system calibration through the bundle adjustment has been shown to be robust, precise, and straightforward. Nevertheless, due to the inherent absence of colour/semantic information in DF images, a significant amount of user input is needed to prepare the image observations for the bundle adjustment. This paper introduces a semi-automated methodology to minimise the amount of user input required to process calibration images and henceforth to facilitate the calibration task. The methodology is optimized for processing images acquired over a custom-made calibration frame with radio-opaque spherical targets. Canny edge detection is used to find distinct structural components of the calibration images. Edge-linking is applied to cluster the edge pixels into unique groups. Principal components analysis is utilized to automatically detect the calibration targets from the groups and to filter out possible outliers. Ellipse fitting is utilized to achieve the spatial measurements as well as to perform quality analysis over the detected targets. Single photo resection is used together with a template matching procedure to establish the image-to-object point correspondence and to simplify target identification. The proposed methodology provided 56,254 identified-targets from 411 images that were used to run a second-time bundle adjustment-based DF system calibration. Compared to a previous fully manual procedure, the proposed methodology has significantly reduced the amount of user input needed for processing the calibration images. In addition, the bundle adjustment calibration has reported a 50\% improvement in terms of image observation residuals.
\end{abstract}

\section{INTRODUCTION}

Biplanar videoradiography (BPVR) or clinically referred to as dual fluoroscopy (DF) imaging systems are increasingly being used to study the in-vivo skeletal biomechanics of human and animal locomotion (Dawson et al., 2011; Kapron et al., 2014; Torry et al., 2011). Using low-dose X-ray radiation, DF systems provide very accurate bone rotation and translation measurements (e.g., $\sim 0.2^{\circ}$ and $0.2 \mathrm{~mm}$ for static imaging and $0.9^{\circ}$ and $0.3 \mathrm{~mm}$ for dynamic imaging) (Anderst et al., 2009). In this research domain, a DF system comprises two X-ray sources, two image intensifiers and two high-speed video cameras. The combination of these elements allows for the stereoscopic imaging of the bones of a joint at high temporal resolution (e.g., 120-250 Hz), from which bone kinematics may be estimated using radiostereometric analysis (e.g., Börlin et al., 2002) or model-based registration approaches (e.g., Bey et al., 2008). DF measurement of skeletal kinematics is based on a few methodological assumptions and needs minimal interaction with the study subject. Following a geometric calibration of the system parameters, DF only requires imaging of a subject's motion within the field-of-view of the X-ray sources while observing the dose limit guidelines. Other motion-capture approaches (i.e., optical and inertial) require substantial interaction with the study subject and sometimes those are inapplicable. Optical motion-capture approaches for example require substantial subject preparation to accurately place skinmounted reflective markers to ensure that rigid body assumptions are met and relevant segment coordinate systems can be established. Further, optical motion-capture approaches suffer from soft-tissue movement artefact, an inherent concern with optical systems due to the relative movement of skin mounted reflective markers with respect to the underlying bone (Akbarshahi et al., 2010; Sharma et al., 2015). Inertial motioncapture approaches on the other hand require a complex integration of three accelerometers, gyroscopes and magnetometers (Roetenberg et al., 2009). These sensors and their power supplies may be contained in a special suit or affixed to the segment under examination. The use of such comparatively bulky measurement units may be problematic in certain populations (e.g., paediatric, injured or pathological) and the lack of a common anatomical coordinate system limits the clinical interpretation of resultant segmental kinematics.

In case of DF imaging, the system calibration can be considered as one of the most challenging tasks, as it needs considerable specialist effort and scientific analysis. The aim of the calibration process is to estimate the system parameters, namely; the cameras' interior orientation and relative orientation parameters (IOPs and ROPs, respectively). The quality of the IOPs and ROPs directly impacts the accuracy of bony measurements. The DF system calibration is conducted prior to any subject testing. It can be achieved by photogrammetric means after capturing multiple images over a grid of radio-opaque beads (i.e., calibration targets) or a perforated metallic sheet (i.e., calibration frame) (Kaptein et al., 2011). Traditional DF calibration approaches are based on two independent steps and do not exploit the benefits of redundant image observations. Typically, these approaches use local models (Ferrigno et al., 2002) or global polynomials (Gutiérrez et al., 2008) to model the distortion parameters (i.e., IOPs) in individual images. This is achieved by measuring the 2D coordinates of the imaged calibration targets and finding their deviations from an idealized location or shape. For the derivation of ROPs, the direct linear transformation (DLT) or its variants are usually used to estimate the position vector and orientation angles of each image (i.e., image exterior orientation parameters, EOPs, which are then used to derive the ROPs). As each image is processed separately, the calibration parameters suffer from high correlations between each other. In addition, 
the derived IOPs vary from one image to another, which is incorrect for a stationary DF system.

Lichti et al. (2015) proposed a rigorous procedure for the calibration of DF systems. Their approach is based on performing bundle adjustment with self-calibration. By simultaneously using redundant measurements of calibration targets in convergent images, it was shown that the solution for the IOPs and ROPs through the bundle adjustment is precise, unique, and straightforward. Nevertheless, to allow for such a bundle adjustment solution, the imaged targets must be manually digitized, spatially measured and then related to their 3D object space homologues. Due to the absence of colour/semantic information in the DF images, a substantial amount of manual work was needed to prepare the input data for bundle adjustment.

The transmissive nature of an X-ray-based imaging approach leads to ambiguous image scenes that are not common in optical photogrammetry. For instance, the calibration targets at different depths may overlap one another. In addition, the targets have no semantic/coded information, which complicates the task of automating the calibration process. This research introduces a framework to facilitate the calibration of DF imaging systems. The paper begins with a review of relevant literature regarding the applications of DF imaging systems and their common calibration approaches. Section 2 provides a brief background on the DF imaging process and summarizes the theoretical bases of the calibration approach proposed by Lichti et al. (2015). Section 3 explains the proposed methodology for the extraction, localization and identification of the calibration targets. The proposed methodology is then evaluated in the results section through a calibration experiment (Section 4). Finally, the paper presents relevant conclusions and recommendations for future work (Section 5).

\section{BACKGROUND}

\subsection{Dual Fluoroscopy Imaging}

In this research, the DF system consists of two timesynchronized X-ray sources (G-1086, Varian, USA), an instrumented treadmill (Bertec, USA), two $406 \mathrm{~mm}$ diameter quad-mode image intensifiers (E5876SD-P2A, Toshiba, Japan), and two high-speed video cameras (DIMAX, PCO, Germany) (Figure 1). The DF imaging process begins with the transmission of an X-ray pulse from the X-ray source, which impinges on the object within the imaging volume. Any radioopaque material in the field-of-view attenuates the incident $\mathrm{X}$ ray pulse, while the un-attenuated $X$-rays continue toward the image intensifier. The intensifier input screen receives the $X$ rays, where they are converted to optical photons and amplified through the intensifier lens mechanism. This conversion results in a green image at the intensifier output screen that is, in turn, recorded as a grey-scale image by the high-speed video camera. In the system under consideration, each camera has a 4 megapixel sensor (i.e., $11 \mu \mathrm{m} \times 11 \mu \mathrm{m}$ pixel size) and the image intensifiers have four different resolution modes. For this testing, an image intensifier resolution of 1.8 line-pairs $/ \mathrm{mm}$ was used. The corresponding DF image pixel resolution was 0.275 $\mathrm{mm}$.

The combination of two X-ray sources, two image intensifiers and two high-speed video cameras provides a means to conduct stereoscopic imaging of objects under clinical and research examination. Briefly, the DF imaging and six-degree-of- freedom reconstruction procedure may be summarised in the following steps:

- Photogrammetric DF system calibration, using a calibration object to determine the cameras interior and exterior orientation parameters (IOPs and ROPs);

- DF image sequence acquisition of bone motions;

- Distortion correction of acquired bone images;

- Acquisition, segmentation and construction of a reference 3D bone model from Computed Tomography (CT) or Magnetic Resonance Imaging (MRI);

- Reconstruction of six-degree-of-freedom bone kinematics using either radiostereometric analysis or model-based registration approaches.

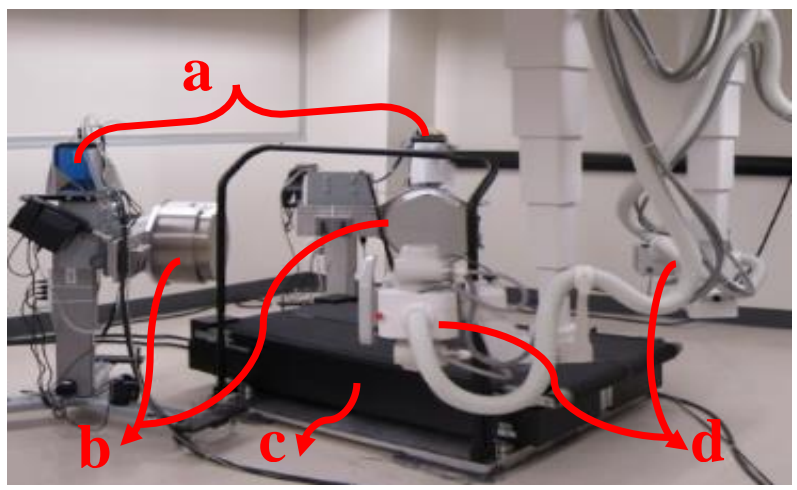

Figure 1. DF imaging system components, (a) high-speed cameras, (b) image intensifiers, (c) treadmill, and (d) X-ray sources

\subsection{Calibration Requirements}

Lichti et al. (2015) proposed a photogrammetric methodology that established the first reference dataset for DF system calibration using bundle adjustment. For detailed description about the mathematical models used and the photogrammetric background on this subject please consult Lichti et al. (2015). Briefly, this methodology used a large number of calibration images and targets and revolved around two main principles, namely: (1) the sensor model definition; and (2) the calibration network configuration. Regarding the sensor model, a single camera, a single image intensifier and a single X-ray source were treated as an ideal pinhole X-ray source model comprising a perspective center (PC) and a flat image plane (Figure 2.a and Figure 2.b).

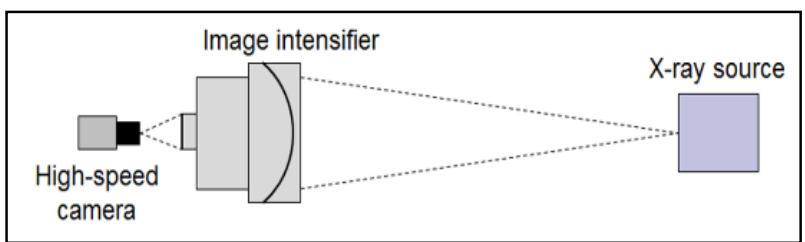

(a)

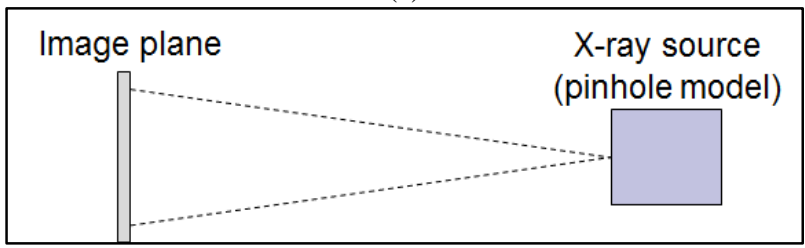

(b)

Figure 2. DF sensor model, (a) actual system configuration and (b) lumped system configuration 
The lumped sensor configuration facilitated the DF system calibration through the bundle adjustment model. Accordingly, the functional task of the bundle adjustment was to estimate the cameras IOPs and ROPs. The IOPs comprised the principal distance $(c)$, the principal point offset $\left(x_{p}, y_{p}\right)$ and a set of additional distortion parameters (i.e., $\mathrm{X}$-ray imaging distortions and the camera distortions). The ROPs described the relative geometric relation between the two cameras and comprised a position vector and three rotation angles.

Regarding the calibration-network configuration, the methodology involved acquiring successive images of a 3D calibration frame through a strong network geometry (Figure 3.a shows an optical image and Figure 3.b shows an X-raybased image of the calibration frame, respectively). To satisfy the geometry requirements (Remondino and Fraser, 2006), the methodology was designed to acquire calibration images from different locations and orientations. It is impractical to relocate the system components in order to acquire convergent images primarily because of the system large mass and the relative orientation parameters between the cameras would be disturbed if moved. Alternatively, the calibration frame was designed to be rotated when collecting the images. A right-handed coordinate system was established at the centroid of the calibration frame (Figure 3.a). By sequentially rotating the calibration frame through $360^{\circ}$ around its $\mathrm{X}, \mathrm{Y}$, and $\mathrm{Z}$ axes, a full coverage of the object space was achieved. Please note that the procedure of having a fixed camera and a portable calibration frame is well-known in photogrammetry and is equivalent to having a fixed calibration frame and a portable camera (Fraser, 2012). For precise estimation of the IOPs, a total of 503 calibration targets (spherical steel beads with a diameter of $3.5 \mathrm{~mm}$ ) were distributed in unique patterns over four faces of the calibration frame.

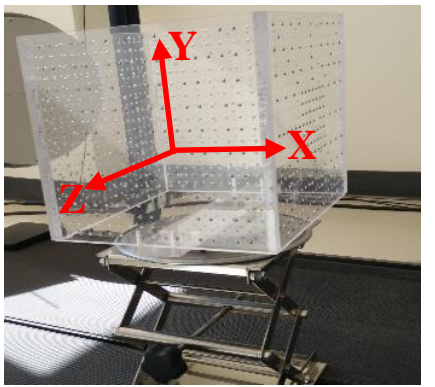

(a)

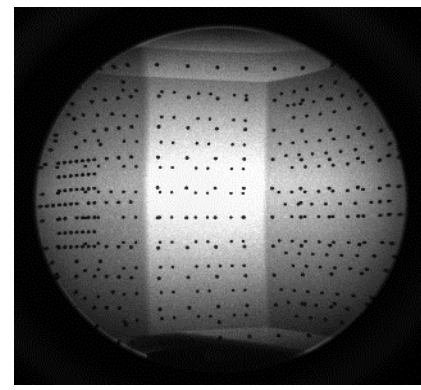

(b)
Figure 3. Calibration frame images, (a) optical image and (b) $\mathrm{X}$-ray-based image

The previous methodology of processing the calibration images involved a manual digitization of all the targets in 300 images (Lichti et al., 2015). The Hough transform (Hough, 1962) was used to determine an initial target location measurement that was refined by an iterative ellipse fitting or circle fitting. This procedure required an average of 15 minutes to process each calibration image. Since the calibration methodology requires the use of a large number of images, a significant user input was needed. This has raised the necessity for a more automated procedure for preparing the calibration data (i.e., the bundle adjustment inputs). The following section illustrates the proposed methodology for processing the calibration images.

\section{TARGETS EXTRACTION, LOCALIZATION, AND IDENTIFICATION}

Spherical targets have been widely used in high-accuracy photogrammetric applications for the calibration purpose (e.g., Sun et al., 2016). Usually, the calibration targets will have unique semantic and geometric properties (i.e., signalized targets) to allow for their automatic extraction/identification as well as to facilitate tasks of spatial measurement in 2D (e.g., Habib et al., 2013; Lari et al., 2014; Tommaselli et al., 2014). In the case of DF images, the calibration targets appear as black circular-like (i.e., an ellipse with low eccentricity) footprints with no semantic information, which complicates the task of automating the bundle adjustment calibration. The methodology of processing the calibration images has three main components, namely; target extraction, target localization, and target identification. The target extraction aims at detecting the targets and to filter out possible outliers. The target localization focuses on achieving the spatial measurements in 2D (i.e., target centroid) and performing quality analysis over the detected targets. The target identification aims at relating the $2 \mathrm{D}$ measurements to their 3D object space homologues (i.e., assigning proper ID for each target).

For the target extraction, Canny edge detection (Canny,1986) is used to find distinct structural components within the images. Edge linking is used to combine the detected edge-pixels in individual groups. And principal components analysis (PCA; Pauly et al., 2002) is applied to detect the targets and to filter out possible outliers. For the target localization, ellipse fitting (Halır and Flusser, 1998) is used to estimate the target centroid and to perform the quality analysis over the detected targets. For the target identification, a quaternion-based single photo resection (SPR; Mazaheri and Habib, 2015) is used together with a template-matching procedure to establish the image-toobject point correspondence.

\subsection{Target Extraction}

Due to the low structural complexity within the calibration images (Figure 3.b), rapid transitions in grey values mainly exist at the targets boundaries. Such variation exhibits a local maximum gradient magnitude that will allow location of the edge pixels through the Canny algorithm. A gradient magnitude profile through four sample calibration targets (Figure 4) demonstrates that among all the pixels within the profile line, the targets edge pixels have a significantly high gradient magnitude. Accordingly, a relaxed gradient threshold is sufficient to detect all the targets within a calibration image through the Canny operator (e.g., edge-pixels with a gradient magnitude value greater than 20), as demonstrated in the edge detection result of a sample calibration image (Figure 5).

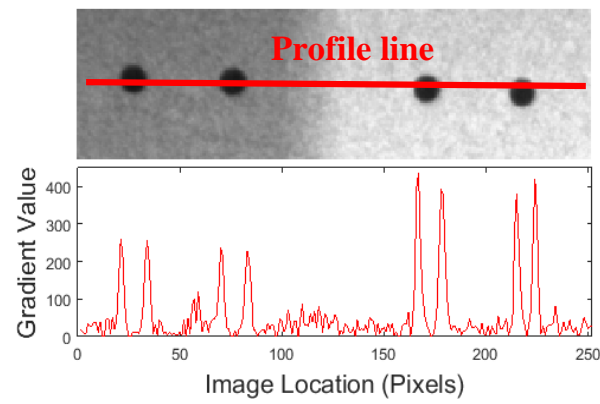

Figure 4. Gradient magnitude profile of four calibration targets (absolute values of gradient magnitude are displayed) 


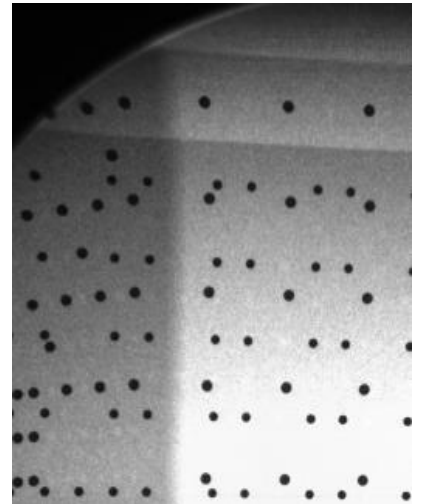

(a)

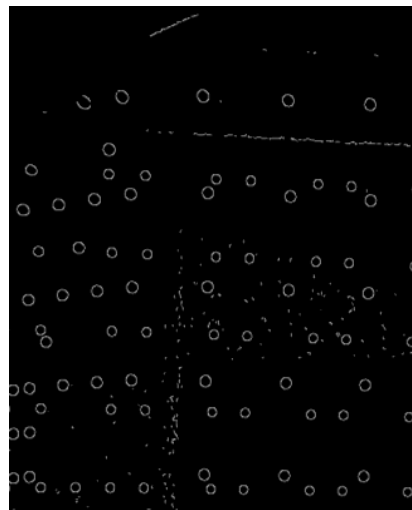

(b)

Figure 5. Canny edge detection sample, (a) the original calibration image and (b) the corresponding extracted edges with a gradient magnitude value greater than 20

Following the edge detection, an edge linking step is applied to separate the edge pixels into individual groups. The edge linking works by tracking a sequence of connected edge pixels. Eventually, the edge pixels that belong to an un-occluded target will result in a circular-like shape (Figure 6.a). Since the 3D calibration frame has four sides containing targets, some targets from different sides might overlap one another. The detected edge pixels from overlapping targets are oval-like shapes (Figure 6.b). Also, areas such as the target field edges feature high image gradients and lead to linear-like shapes (Figure 6.c). Therefore, PCA (Equation 1) is used to automatically determine the geometric shapes of the grouped edge pixels. Through the PCA, the circular-like shapes are kept and are hypothesised to be potential targets, while the other shapes (i.e., linear-like, or oval-like) are filtered out since those don't serve the calibration process. The application of PCA over the covariance matrix of a group of edge pixels results in two eigenvalues (i.e., $\lambda_{1}$, and $\lambda_{2}$ ). For circular-like shapes (Figure 6.a), the two eigenvalues will be almost equivalent (e.g., $\lambda_{1} / \lambda_{2} \geq 0.70$ ). For linear-like or oval-like shapes (Figure $6 . \mathrm{b}$ and c), one of the eigenvalues will be much larger than the other (e.g., $\lambda_{1} / \lambda_{2}<0.70$ ). Please note that in some incidences the targets might be partially visible and would pass the PCA step. These are later filtered out by evaluating the quality of ellipse fitting as will be explained in the following section.
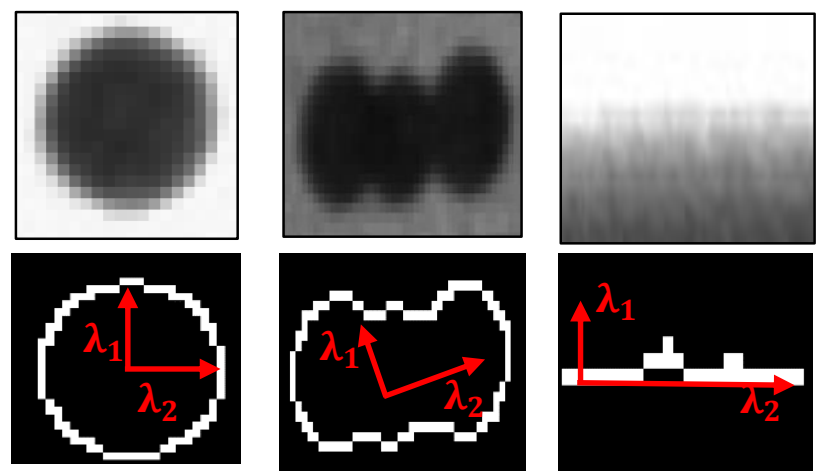

(a)

(b)

(c)

Figure 6. PCA of edge pixels, (a) circular-like shape, (b) ovallike shape and (c) linear-like shape

$$
\begin{gathered}
C_{2 \times 2}=\left[\begin{array}{ll}
\vec{e}_{1} & \vec{e}_{2}
\end{array}\right]\left[\begin{array}{cc}
\lambda_{1} & 0 \\
0 & \lambda_{2}
\end{array}\right]\left[\begin{array}{l}
\vec{e}_{1}^{T} \\
\vec{e}_{2}^{T}
\end{array}\right] \\
\text { where: } C_{2 \times 2}=\frac{1}{n} \sum_{i=1}^{n}\left(\left[\begin{array}{l}
P_{x_{i}} \\
P_{y_{i}}
\end{array}\right]-\left[\begin{array}{l}
\bar{P}_{x} \\
\bar{P}_{y}
\end{array}\right]\right)\left(\left[\begin{array}{l}
P_{x_{i}} \\
P_{y_{i}}
\end{array}\right]-\left[\begin{array}{l}
\bar{P}_{x} \\
\bar{P}_{y}
\end{array}\right]\right)^{T} 1 \\
\text { and, } \bar{P}=\frac{1}{n} \sum_{i=1}^{n}\left[\begin{array}{l}
P_{x_{i}} \\
P_{y_{i}}
\end{array}\right]
\end{gathered}
$$

where $C$ is the covariance matrix, $\left(\vec{e}_{1}, \vec{e}_{2}\right)$ and $\left(\vec{\lambda}_{1}, \vec{\lambda}_{2}\right)$ are the eigenvectors and the eigenvalues, respectively, $\left(P_{x_{i}}, P_{y_{i}}\right)$ is the $i^{\text {th }}$ pixel coordinate, and $\left(\bar{P}_{x}, \bar{P}_{y}\right)$ is the centroid of the grouped edge pixels.

\subsection{Target Localization}

The DF system calibration requires precise and accurate 2D measurements of the centres of imaged targets. These measurements serve as the observations needed for the bundle adjustment. Theoretically, the perspective projection of a spherical target leads to an elliptical shape in the image space (Luhmann, 2014). The application of ellipse fitting over the target edge-pixels provides a reliable 2D measurement of its centre. The ellipse fitting method proposed by Halır and Flusser (1998) is used here as it has been shown to be accurate and numerically stable. This method considers a general-conic model (Equation 2) and performs a non-iterative least squares adjustment. Following the estimation of the ellipse parameters, the ellipse centre is derived according to Equation 3. Figure 7 shows an example of ellipse fitting result from a set of target edge pixels. As can be seen, the estimated ellipse precisely matches the target edge pixels.

In general, a high quality of fit results when dealing with a fully visible target (e.g., $0.25 \pm 0.10$ pixel RMS error of fit). In contrast, a low quality of fit results for partially visible or blurred targets (e.g., roughly $0.60 \pm 0.10$ pixel RMS error of fit) (Figure 8). Such targets lead to low quality image measurements that negatively affect the bundle adjustment solution. Therefore, the detected targets are evaluated according to their quality of fit. The targets associated with significantly high RMS error value are filtered out (e.g., targets with RMS error of fit greater than 0.5 pixel).

$$
\begin{gathered}
f(x, y)=A x^{2}+B x y+C y^{2}+D x+E y+F=0 \\
x_{c}=\frac{2 C D-B E}{B^{2}-4 A C} \\
y_{c}=\frac{2 A E-B D}{B^{2}-4 A C}
\end{gathered}
$$

where $A, B, C, D, E$, and $F$ are the ellipse parameters, and $\left(x_{c}, y_{c}\right)$ are the coordinates of the target centre.

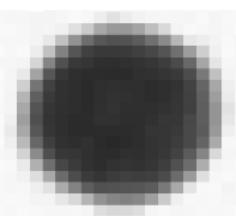

(a)

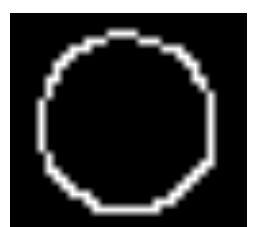

(b)

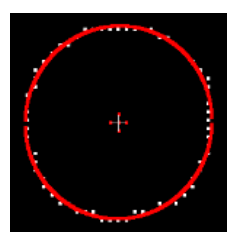

(c)
Figure 7. Ellipse fitting sample over fully visible target, (a) source image, (b) target edge pixels and (c) the fitted ellipse 


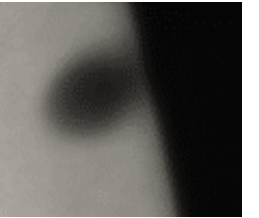

(a)

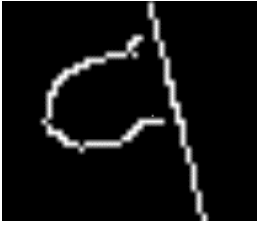

(b)

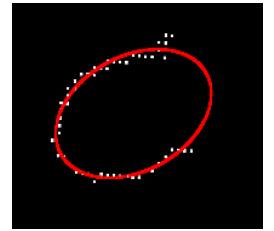

(c)

Figure 8. Ellipse fitting sample over partially visible target, (a) source image, (b) target edge pixels and (c) the fitted ellipse

The ellipse fitting provides reliable 2D measurements that are necessary to run the bundle adjustment. Nevertheless, one should bear in mind the eccentricity error that is inherent to the measurements of spherical targets (Luhmann, 2014). In the case of optical imaging, the perspective projection of a spherical target leads to an ellipse with low eccentricity (circular-like shape). Based on its diameter and the angle of projection, the target will possess varying eccentricity values. As a result, the target centre estimate in 2D might not accurately represent its 3D shape, which degrades the bundle adjustment output. In the case of DF imaging, it was observed that the targets near to the image bounds will possess high eccentricity (e.g., Figure 8.a). The DF imaging mechanism involves two projection incidences (Figure 2.a); first, the targets are projected onto the hemispherical intensifier input screen. Second, the intensifier output screen display is projected onto image space through the camera perspective centre. The projection of the calibration targets onto the hemispherical input screen maximises the targets eccentricity. A geometric simulation for the DF system to evaluate the impact of eccentricity on the target measurements is currently in progress. A preliminary experiment has yielded a maximum error of 1 pixel in the target measurements due to the eccentricity. At present, we assume that the measurements from ellipse fitting are adequate to establish a sufficient base for the DF system calibration. We agree with Luhman (2014) that the eccentricity error will be partially compensated by the radial lens distortion parameters. Future work will focus on establishing a realistic estimation/compensation for the eccentricity related error. The following section introduces the proposed procedure for target identification.

\subsection{Target Identification}

One of the fundamental tasks that must be done prior to the bundle adjustment is to relate the $2 \mathrm{D}$ measurements to their $3 \mathrm{D}$ homologues. Typically, the targets will have unique IDs/nametags and all the $2 \mathrm{D}$ measurements that belong to a particular target will be given the same ID. This is essential to establish the attributes needed for the bundle adjustment (Fraser and Edmundson, 2000). In this work, the targets are distributed in unique patterns over the four sides of the calibration frame to simplify their visual identification. However, due to the large number of targets/images that are used for the calibration process, a significant amount of time is required to manually identify each individual target in all images. Also, the absence of semantic/colour information as well as the crowded images (with targets) might lead to incorrect-identification incidences. Given that the images of the calibration frame are sequentially acquired (Figure 9), a high degree of overlap between the successive images results. Here, we introduce a two-step methodology to simplify target identification and to reduce the amount of required manual work. First, an SPR-based procedure is used to identify the targets in a particular image (e.g., the first image in a sequence). Second, a template-matching procedure is used to inherit the IDs between the conjugate targets in

successive images. The SPR-based procedure is performed by manually identifying a minimum of three well-distributed targets within an image (Figure 10). The 2D coordinates of the targets, their 3D homologues and nominal values of IOPs are used to estimate the image EOPs through the SPR. After the estimation of the EOPs, the 3D coordinates of all the targets are projected into image space using the collinearity equation (Granshaw, 1980). The projected targets will fall near to their image homologues (Figure 11). Since the 3D coordinates of all the targets are associated with unique IDs, the ID of each projected target can be assigned to its nearest image measurement. Once all targets in a given image have been identified, they are used as a template to identify their conjugates in the following image in the sequence. The template targets are shifted in order to achieve a good alignment with their conjugates in the second image (Figure 12). Once the alignment is achieved, the IDs are transferred from the template targets to their conjugates. The process of loading a template from an image to the following image is repeated for the entire images sequence. Please note that in a successive pair of images the targets from different sides of the calibration frame move in opposite directions relative to the applied rotation (e.g., the arrows directions in images 2 and 3 - Figure 9). Therefore, multiple templates are usually generated to simplify the alignment process. Each template contains the targets of an individual side of the calibration frame. This provides more freedom to the user to move templates of different sides in opposite directions simultaneously. The template-matching procedure requires little manual effort and provides accurate target identification even for images crowded with many targets. Due to the high overlap between the successive images, the conjugate targets will have only small variations in their $2 \mathrm{D}$ locations. A few targets might appear/disappear between two successive images. The new appearing targets will not have template conjugates, so those have to be manually identified. The user can simply drag their IDs from a previously projectedtargets.

The selected targets for SPR should be well-separated for a reliable estimation of the EOPs (e.g., the distribution of the targets 1, 2 and 3 in Figure 10). In addition, the selected targets must be non-collinear since the collinear ones are insufficient to estimate all the rotations of the EOPs. The quality of the EOPs estimated by the SPR plays an essential role in determining the closeness/alignment between the projected targets and their image homologues. The existence of high distortion will also affect the alignment. The quality of the EOPs is highly dependent on the used IOPs as well as the distribution/number of the selected targets. IOPs from a previous system calibration (i.e., $x_{p}, y_{p}$ and $c$ ) are used for the SPR. Since the IOPs are dependent on the DF system setup, they may vary significantly between calibrations. Accordingly, the user might need to select more than three targets (e.g., five) to achieve higher quality EOP estimates. The user should cumulatively select targets for SPR and then evaluate the estimated EOPs relative to the alignment between the projected targets and their image homologues. The unique target pattern distributions of the four sides of the calibration frame help the user to visually confirm an appropriate alignment. Once the alignment is achieved (e.g., Figure 11), the user can transfer the IDs from the projected targets to their image homologues. Due to occlusions, usually there will be more projected targets than image targets. Therefore, the ID transfer is done based on a distance threshold from the image targets to the projected target (e.g., the image target will get the ID of the closest projected target within a set distance, in this case 50 pixels). 


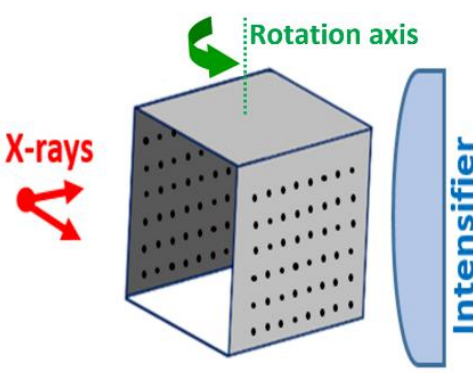

Imaging mechanism

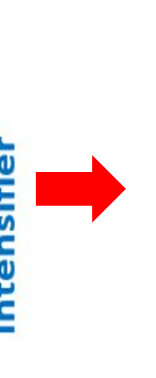

Figure 9. Imaging mechanism and sample of successive images

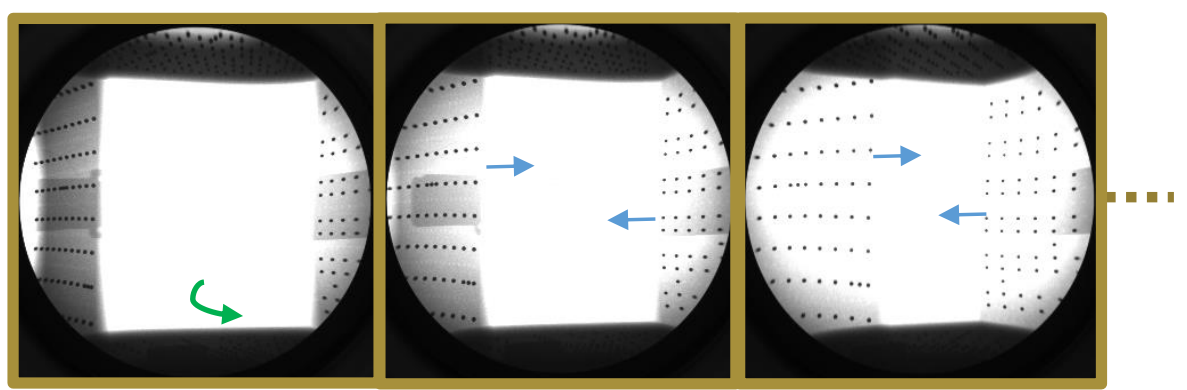

image 3

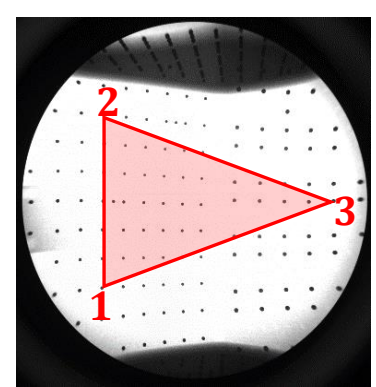

Figure 10. Distribution of selected three targets for SPR

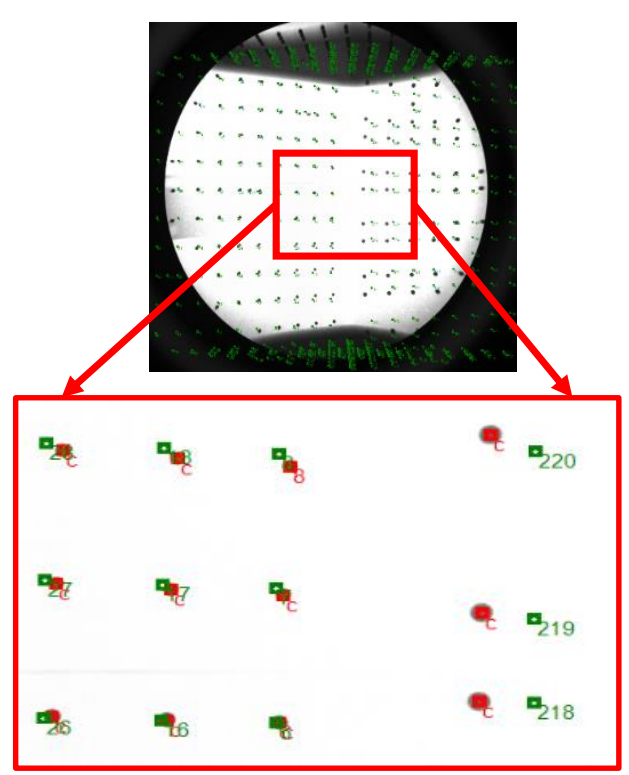

Figure 11. Projection of the targets 3D coordinates onto a calibration image (projected targets in green and the image measurements in red)

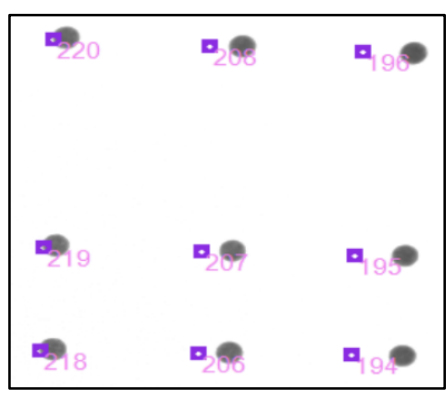

Figure 12. Template targets (in magenta) aligned over their conjugates
Some images are crowded with targets (e.g., the image in Figure 3.b), which might lead to a scenario where a projected target lies within a similar distance to two image targets. To deal with this situation, a simple routine is used to pinpoint the possible ambiguities in identification. Then the user has the manual freedom to drag and drop the IDs from the projected targets to their image homologues based on the visual evaluation.

\section{EXPERIMENTAL RESULTS AND DISCUSSION}

\subsection{Calibration Data Collection}

A DF system calibration session was performed in June, 2015 at the Clinical Movement Assessment Laboratory, University of Calgary, Alberta, Canada. The system was setup in a typical configuration for kinematic analysis of bone movements. The calibration frame was placed in a central location between the $\mathrm{X}$-ray sources and the image intensifiers. It was mounted on height adjustable turntable base to allow its rotation when the system was activated. Preliminary exposures were obtained to ensure that the calibration frame was visible to both intensifiers. The system was set to acquire images at $6 \mathrm{~Hz}$ using both cameras. Three orientation configurations were applied to fully cover the calibration frame from all sides. For the first orientation, the calibration frame was placed such that its $\mathrm{X}$-axis pointed in the vertical upwards direction. Then, the calibration frame was manually rotated about the $\mathrm{X}$-axis through $360^{\circ}$ while the system was activated. The calibration frame was relocated, and a similar procedure was followed for the other two rotation configurations having the $\mathrm{Y}$ and $\mathrm{Z}$ axes each pointing in the vertical upwards direction, respectively.

\subsection{System Calibration through Bundle Adjustment}

The proposed methodology provided 56,268 identified-targets from 411 images that were used to conduct a second-time bundle adjustment-based system calibration. The objective of the calibration experiment was to evaluate the quality of derived image measurements and to perform analysis over a calibration model. The target extraction/localization was completely automatic and required an average processing time of 1 second per image. The target identification task required an average manual interaction of $\sim 3$ minutes per image. Compared to the previous experiment of processing calibration images (Lichti et al., 2015), the proposed procedure has led to $80 \%$ reduction in terms of manual processing time. The template matching was very useful to inherit the IDs over the conjugate targets in the overlapping images. 
The calibration experiment was performed using the bundle adjustment calibration model proposed in Lichti et al. (2015). A total of 34 parameters were used for the modelling of the IOPs of each lumped imaging system. Specifically, $x_{p}, y_{p}$ and $c$ in addition to $31 \mathrm{X}$-ray-imaging and optical-imaging distortion parameters. The values of $x_{p}, y_{p}$ and $c$ from the previous calibration were used as initial approximations for the iterative bundle adjustment solution and other distortion parameters were initialized to zeros. The initial approximations of each image's EOPs were obtained from the SPR. The bundle adjustment with self-calibration was performed with the free-network datum definition. Table 1 lists the number of images and targets used, the degrees of freedom as well the output root mean square (RMS) of image observation residuals.

Table 1. Bundle adjustment parameters

\begin{tabular}{|c|c|c|c|c|c|}
\hline \multirow{2}{*}{ 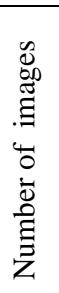 } & \multirow{2}{*}{ 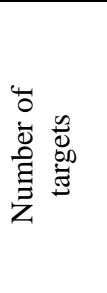 } & \multirow{2}{*}{ 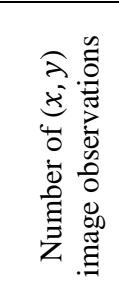 } & \multirow{2}{*}{ 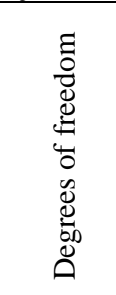 } & \multicolumn{2}{|c|}{$\begin{array}{c}\text { RMS of image } \\
\text { observation residuals } \\
\text { (pixel) }\end{array}$} \\
\hline & & & & 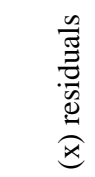 & $\begin{array}{l}\frac{n}{\tilde{J}} \\
\stackrel{0}{\Xi} \\
\stackrel{0}{0}\end{array}$ \\
\hline 411 & 56,254 & 112,508 & 108,472 & 0.25 & 0.27 \\
\hline
\end{tabular}

The proposed methodology was a reliable tool to provide the image measurements needed for the DF system calibration. Reasonable values of RMS image observation residuals (Table 1) were achieved (i.e., a precision of almost a 1/4 pixel). As a second-time bundle adjustment-based system calibration, the $1 / 4$ of a pixel value counts as a promising result and a solid basis to continue toward a fully automated system calibration. For the calibration of digital-cameras in optical close-range photogrammetry, a value of $1 / 20$ of a pixel or better is achievable currently (Fraser, 2012). This precision can be achieved for the calibration of well-defined camera models while using signalized targets, which is not the case for the DF system. Several factors are speculated to be temporarily preventing achievement of better RMS. For instance, the aforementioned eccentricity-related error might be a factor. Also, the DF imaging involves empirical models to describe the many physical sources of systematic error behaviour. Compared to the previous system calibration, a $50 \%$ improvement was achieved in terms of RMS image observation residuals. The previous experiment (Lichti et al., 2015) has a similar configuration to the one reported here and resulted in $\sim 1 / 2$ of a pixel RMS image observation residuals. The previous experiment was based on manual digitization of all the calibration targets. The Hough transform was used in the previous experiment to determine an initial target centre followed by an iterative ellipse fitting or circle fitting to refine the initial centre.

Table 2 lists the estimated IOPs of each lumped imaging system for the current setup. For space management, only the $x_{p}, y_{p}$, and $c$ estimates are shown. The values of $\left(x_{p}, y_{p}\right)$ are far from an idealized $(1008,1008)$ principal point. This is due to an imperfect alignment between the system imaging components.

Table 2. Imaging system IOPs

\begin{tabular}{|l|c|c|c|}
\hline & $x_{p}$ (pixels) & $y_{p}$ (pixels) & $c$ (pixels) \\
\hline Cam1 & 1078.19 & 1104.67 & 5495.25 \\
\hline Cam2 & 860.50 & 1306.67 & 5605.75 \\
\hline
\end{tabular}

Figure 13 shows the estimated image positions and the 3D coordinates of the calibration frame. The network provides a strong geometric configuration of calibration images. For completeness, please note that a few images - other than the 411 images reported here - were successfully processed using the proposed methodology but were not included in the bundle adjustment calibration. The used bundle adjustment software employs the traditional three Euler angle rotation parametrization (i.e., $\omega, \varphi, \kappa)$. The excluded images from the calibration process had a secondary rotation $(\varphi)$ angle close to $90^{\circ}$ or $270^{\circ}$. These angle values are known to cause the Gimballock condition and therefore are excluded from the calibration. A quaternion-rotation-based bundle adjustment would have been useful to include these images for the calibration process and will be implemented in future work.

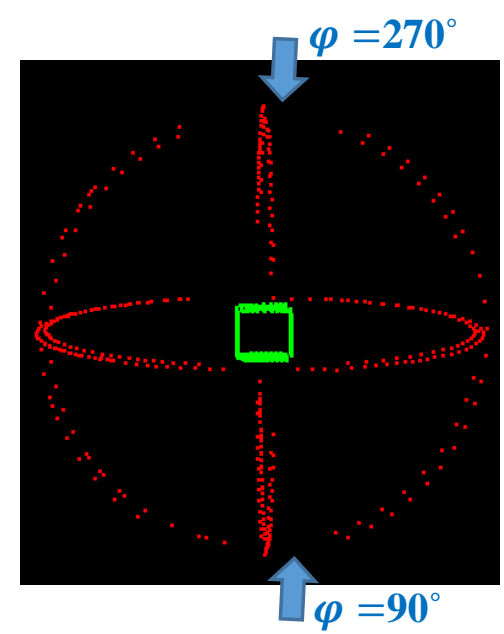

Figure 13. A 3D display of the image positions (in red) and the calibration-targets (in green)

\section{CONCLUSIONS AND FUTURE WORK}

This paper presented a framework to facilitate the calibration of DF imaging systems. An automated procedure was introduced for the detection and localization of calibration targets. A twostep procedure was introduced to simplify the target identification task and to reduce the amount of manual work required in this regard. The proposed methodology was able to successfully provide reliable inputs for a DF system calibration experiment. The proposed methodology led to a substantial reduction in terms of the time needed for processing the calibration images. The bundle adjustment calibration using the derived image measurements resulted in $\sim 1 / 4$ of a pixel RMS image observation residuals, which forms a $50 \%$ improvement compared to a recently achieved value. This is considered as a promising result for the calibration of an X-ray-based imaging system and a solid basis to continue toward a fully automated DF system calibration. The RANdom Sample consensus (RANSAC) method will be tested to automate the SPR step. We will investigate the possibility of using optical flow computations to achieve the template matching procedure. Other calibration-frame shapes or target distributions will be considered as well. Future work will focus on improving the target measurement accuracy by considering the eccentricity related errors. Other calibration concerns such as a better modelling of the system parameters is also under consideration. 


\section{ACKNOWLEDGEMENTS}

This work was supported by Alberta Innovates Technology Futures (AITF) fund and the Natural Science and Engineering Research Council of Canada (NSERC).

\section{REFERENCES}

Akbarshahi, M., Schache, A.G., Fernandez, J.W., Baker, R., Banks, S., Pandy, M.G., 2010. Non-invasive assessment of softtissue artifact and its effect on knee joint kinematics during functionalactivity. J Biomech, 43(7), pp. 1292-1301.

Anderst, W., Zauel, R., Bishop, J., Demps, E., Tashman, S., 2009. Validation of three-dimensional model-based tibiofemoral tracking during running. Med Eng Phys, 31(1), pp. 1016.

Bey, M.J., Kline, S.K., Tashman, S., Zauel, R., 2008. Accuracy of biplane x-ray imaging combined with model-based tracking for measuring in-vivo patellofemoral joint motion. J Orthop. Surg, 3:38.

Börlin, N., Thien, T., Kärrholm, J., 2002. The precision of radiostereometric measurements Manual vs. digital measurements. J Biomech, 35(1), pp. 69-79.

Canny, 1986. A computational approach to edge detection. IEEE Trans Pattern Anal. Mach. Intell, 8-PAMI, pp. 679- 698.

Dawson, M.M., Metzger, K.A., Baier, D.B., Brainerd, E.L., 2011. Kinematics of the quadrate bone during feeding in mallard ducks. J Exp Biol, 214(12), pp. 2036-2046.

Ferrigno, G., Borghese, Forlani, C., Cerveri, P., 2002. Distortion correction for $\mathrm{X}$-ray image intensifiers: local unwarping polynomials and RBF neural networks. Med Phys, 29(8), pp. 1759-1771.

Fraser, C., 2012. Automatic camera calibration in close-range photogrammetry. In: Proc. ASPRS, Sacramento,CA, USA.

Fraser, C.S., Edmundson, K.L., 2000. Design and implementation of a computational processing system for offline digital close-range photogrammetry. ISPRS J Photogramm Remote Sens, 55(2), pp. 94-104.

Granshaw, S., 1980. Bundle adjustment methods in engineering photogrammetry. Photogramm Rec. 10(56), pp. 181-207.

Gutiérrez, L.F., Ozturk, C., McVeigh, E.R., Lederman, R.J., 2008. A practical global distortion correction method for an image intensifier based x-ray fluoroscopy system. Med Phys, 35(3), pp. 997-1007.

Habib, A., Lari, Z., Kwak, E., Al-Durgham, K., 2013. Automated detection, localization, and identification of signalized targets and their impact on digital camera calibration. Braz J Cartogr RBC - Rev Bras Cartogr A, 65(4), pp.785-803.

Halır, R., Flusser, J., 1998. Numerically stable direct least squares fitting of ellipses. In: Proc. 6th International Conference in Central Europe on Computer Graphics and Visualization. WSCG. Citeseer, pp. 125-132.

Hough, P.V.C, 1962. Method and Means for recognizing complex patterns. US Patent No. 3069654.
Kapron, A.L., Peters, C.L., Maas, S.A., Bey, M.J., Zauel, R., Anderson, A.E., 2014. Accuracy and Feasibility of dual fluoroscopy and model-based tracking to quantify in vivo hip kinematics during clinical exams. J Appl Biomech, 30(3), pp. 461-470.

Kaptein, B.L., Shelburne, K.B., Torry, M.R., Erik Giphart, J., 2011. A comparison of calibration methods for stereo fluoroscopic imaging systems. J Biomech, 44(13), pp. 25112515 .

Lari, Z., Habib, A., Mazaheri, M., Al-Durgham, K., 2014. Multi-camera System Calibration with Built-in Relative Orientation Constraints (Part 1) Theoretical Principle. J Korean Soc Surv Geod Photogramm Cartogr, 32(3), pp. 191-204.

Lichti, D.D., Sharma, G.B., Kuntze, G., Mund, B., Beveridge, J.E., Ronsky, J.L., 2015. Rigorous Geometric Self-Calibrating Bundle Adjustment for a Dual Fluoroscopic Imaging System. IEEE Trans Med Imaging, 34(2), pp. 589-598.

Luhmann, T., 2014. Eccentricity in images of circular and spherical targets and its impact on spatial intersection. Photogramm Rec, 29(148), pp. 417-433.

Mazaheri, M., Habib, A., 2015. Quaternion-Based Solutions for the Single Photo Resection Problem. Photogramm Eng Remote Sens, 81(3), pp. 209-217.

Remondino, F., Fraser, C., 2006. Digital camera calibration methods: considerations and comparisons. Int Arch Photogramm Remote Sens Spat Inf Sci, 36(5), pp. 266-272.

Roetenberg, D., Luinge, H., Slycke, P., 2009. Xsens MVN: full 6DOF human motion tracking using miniature inertial sensors. Xsens Motion Technol. BV Tech Rep.

Sharma, G.B., Kuntze, G., Kukulski, D., Ronsky, J.L., 2015. Validating Dual Fluoroscopy System Capabilities for Determining In-Vivo Knee Joint Soft Tissue Deformation: A Strategy for Registration Error Management. J Biomech. 48(10), pp. 2181-2185.

Sun, J., He, H., Zeng, D., 2016. Global Calibration of Multiple Cameras Based on Sphere Targets. Sensors, 16(1), 77.

Tommaselli, A.M.G., Marcato Jr., J., Moraes, M.V.A., Silva, S.L.A., Artero, A.O., 2014. Calibration of panoramic cameras with coded targets and a $3 \mathrm{~d}$ calibration field. ISPRS Int Arch Photogramm Remote Sens Spat Inf Sci, XL-3/W1, pp. 137142.

Torry, M.R., Shelburne, K.B., Peterson, D.S., Giphart, J.E., Krong, J.P., Myers, C., Steadman, J.R., Woo, S.L.-Y., 2011. Knee kinematic profiles during drop landings: A biplane fluoroscopy study. Med Sci Sports Exerc, 43(3), pp. 533-541. 